\title{
EL ECOFEMINISMO EN EL DESARROLLO RURAL SOSTENIBLE ${ }^{1}$
}

\author{
Mario Alexander Cabrera Duarte ${ }^{2}$
}

\begin{abstract}
El ecofeminismo en el desarrollo rural sostenible
Resumen: El ecofeminismo es una valiosa fuente del conocimiento humano que ofrece, desde su teoría y práctica, la oportunidad de contribuir a mejorar las condiciones de vida de las personas. En este artículo exploro la importancia que tiene para fomentar el desarrollo rural sostenible en los países empobrecidos, al evidenciar la urgente necesidad que existe en sus comunidades rurales de respetar a las mujeres y la naturaleza ${ }^{3}$.

Palabras clave: Ecofeminismo, mujeres, desarrollo rural sostenible, países empobrecidos.
\end{abstract}

\section{Ecofeminism in Sustainable Rural Development}

Abstract: Ecofeminism is a valuable source of human knowledge. It offers, from theory to practice, the opportunity to help improve people's lives. In this article, I address the importance of promoting sustainable rural development in poor countries because of the evident lack of respect to women and nature in their rural communities.

Key words: Ecofeminism, women, sustainable rural development, impoverished countries.

“Con la violación de la naturaleza está vinculada la violación y marginación de la mujer, especialmente en el Tercer Mundo." (Shiva 1995: 82)

\section{Ecofeminismo y desarrollo rural sostenible}

El ecofeminismo y el desarrollo rural sostenible son dos categorías de análisis relativamente nuevas, su aparición se puede situar en el último tercio del siglo XX. El primer término ha sido producto del posicionamiento político que van asumiendo las

\footnotetext{
${ }^{1}$ Fecha de recepción: 21/08/2014.

Fecha de aceptación: 14/09/2014.

${ }^{2}$ Docente del Instituto Francisco J. Mejía, así como del Departamento de Ciencias Sociales del Centro Universitario de Educación a Distancia de la Universidad Pedagógica Nacional Francisco Morazán de Honduras. Doctorando en Filosofía en la Universidad de Valladolid y becario del Proyecto PEACE; $\square$ ratioupn@yahoo.es.

${ }^{3}$ El término naturaleza tiene varios significados, en mi caso al utilizarlo hago referencia al propuesto por Jorge Riechmann (2000: 99-100) quien lo define como el sistema organizado de los ecosistemas (biosfera), en donde existe un conjunto de seres vivos y no vivos, que están físicamente constreñidos en un espacio determinado.
} 
mujeres y su preocupación ante los problemas ecológicos que azotan a la humanidad, mientras que el segundo, ha surgido de las iniciativas impulsadas por los Organismos de Cooperación Internacional, las Organizaciones No Gubernamentales -conocidas popularmente como $\mathrm{ONG}-$, los gobiernos y las organizaciones comunitarias que promueven proyectos de desarrollo bajo el ideal de mejorar las condiciones de vida en las áreas rurales, pero sin comprometer la existencia de los recursos naturales que van a necesitar las generaciones futuras.

Según Alicia Puleo (2011: 405) el feminismo aceptó, desde hace más de tres décadas, el desafío de reflexionar sobre la crisis ecológica desde sus propias claves, teniendo como elementos centrales a considerar el patriarcado, el androcentrismo, el sexismo, el cuidado y el género, los que son de utilidad para desvelar la exclusión que sufren las mujeres y la depredación a la que se ve sometida la naturaleza en la sociedad patriarcal. Así, sus diferentes corrientes entre las que se encuentran según Karen Warren (2003: 12) las de tendencia liberal, tradicional, marxista, radical y socialistas, fueron dando origen a lo que hoy se conoce con el nombre de ecofeminismo.

Aunque existe esa variedad de corrientes, en mi caso estoy utilizando el término ecofeminismo de forma general, entendiéndolo como el movimiento de creación de conocimiento y desarrollo de una práctica social, orientada a concientizar a las personas de los problemas que sufren las mujeres -en especial por su condición de género- y de la crisis ecológica que estamos viviendo. El ecofeminismo es reflexión teórica, práctica social y utopía, sus acciones están encaminadas a construir sociedades que sean capaces de respetar a las mujeres y la naturaleza.

Tomando de referencia a Maria Mies y Vandana Shiva (1997: 25-26) se puede expresar que aunque el término ecofeminismo fue acuñado por Françoise d'Eaubonne a finales de 1970 y principio de 1980, como saber es antiguo. Además las mismas autoras evidencian que desde la década del 70 surgió con una fuerte práctica social en contra de la destrucción del ambiente.

Actualmente a nivel mundial, tanto en las principales ciudades de los países empobrecidos como en las comunidades rurales aledañas a ellas, las organizaciones 
feministas luchan por construir un mundo en donde florezca la vida. Voces como las de Vandana Shiva de la India y Wangari Maathai de Kenia, han sido una guía y servido de inspiración por el trabajo desarrollado, lo que se ha reconocido a nivel internacional, a través de instituciones como las Naciones Unidas y la Fundación Alfred Nobel. ${ }^{1}$

El ecofeminismo con sus acciones de manera consciente o inconsciente, fomenta la búsqueda de un desarrollo sostenible, el que es contrario al modelo de desarrollo liberal patriarcal, ya que este último se ha enfocado en maximizar las ganancias, reducir costos y acumular en unas pocas manos los beneficios que genera la producción económica, a costa del dolor infligido a millones de seres humanos.

En el modelo de desarrollo liberal patriarcal, las mujeres son las principales víctimas, ${ }^{2}$ especialmente en los países empobrecidos, donde hay débiles legislaciones para proteger a la población de los daños causados por las empresas mineras, hidroeléctricas, petroleras, las dedicadas a los monocultivos a gran escala, las forestales y las que experimentan con Organismos Genéticamente Modificados, entre otras.

El ecofeminismo al fomentar el empoderamiento de las mujeres y la defensa de la naturaleza, se convierte en un movimiento vital para impulsar el desarrollo rural sostenible, debido a los puntos de coincidencia que existen entre ambos; ya que este último busca "el mejoramiento integral del bienestar social de la población” (Congreso de la Unión 2001: 2) que habita en las zonas no urbanas. Lo que implica prestarle atención al uso racional de los recursos naturales y a la construcción de sociedades que sean capaces de eliminar toda forma de opresión que esté presente o pueda surgir entre hombres y mujeres.

\footnotetext{
${ }^{1}$ Vandana Shiva recibió en 1993 el Premio Global 500 del Programa de las Naciones Unidas para el Medio Ambiente, mientras que Wangari Maathai fue galardonada con el Premio Nobel de la Paz en el 2004.

${ }^{2}$ Uno de los tantos ejemplos sobre los efectos del modelo de desarrollo liberal patriarcal en las comunidades rurales, se encuentra en el caso de la explotación petrolera que realizó la Empresa Texaco en Ecuador, durante los años 1964 y 1992. Martín Beristain, Carlos, Darío Páez Rovira e Itziar Fernández (2009: 166167) narran en su libro "Las palabras de la selva" que las mujeres fueron más insistentes en denunciar los daños provocados a su modo de vida y la naturaleza; haciendo énfasis en los problemas de salud que les generó a nivel personal y familiar; los causados a la vida comunitaria producto del alcoholismo, la mayor disponibilidad de dinero para los hombres, la intimidación sufrida por la presencia militar, la intensificación de su rol de cuidadoras, así como los abusos y violencia sexual a la que se expusieron o sufrieron.
} 
Cuando me refiero al desarrollo rural sostenible lo hago desde la dimensión teórica, la que lo entiende según Leonard Field (1997: 31-32) como un proceso de mejora del bienestar social, cimentado en la necesidad de no alterar las condiciones fundamentales de la vida humana - por ejemplo la capa de ozono y las fuentes de agua-; de contar con una base estable o creciente de recursos primarios; de mejorar la producción, distribución de bienes y servicios, así como reconocer la existencia de valores intrínsecos no utilitarios.

\section{El rol social de las mujeres rurales}

El entorno cultural en que nacemos determina en gran medida nuestra forma de ser y de pensar, nos construye a través de "una serie de mandatos y modelos, por un mundo de lo simbólico en el que estamos todos inmersos" (Puleo 2013: 5), del que es difícil escapar, en especial cuando se pertenece a comunidades pequeñas, aisladas de los grandes polos de desarrollo, con una presencia marginal de la acción estatal y con miembros que pertenecen a culturas minoritarias.

Las personas modeladas por el entorno cultural en que viven, se encargan de mantenerlo en funcionamiento, aun siendo víctimas del mismo, como lo reconoce Gebara (2000: 22) con la dominación y exclusión de las mujeres en las estructuras androcéntricas, las que se mantienen y reproducen en cierta medida, por la complicidad femenina que adquiere su rostro más visible en la obediencia.

Esa complicidad a la que se refiere Gebara, no debe ser motivo para condenar a las mujeres, debido a que ellas lo hacen respondiendo a la fuerza de la tradición, ${ }^{3}$ a la ideología que se ha enraizado en sus conciencias, a la ignorancia o por temor. En las comunidades rurales, los roles sociales se encuentran muy marcados a partir de la condición sexual de la persona, la esfera del hogar es responsabilidad de una ama de casa, que debe dar cuentas y estar subordinada al jefe del hogar, quien toma las decisiones en el interior de la familia y la representa a nivel comunitario.

\footnotetext{
3 Calatrava Requema (2002: 74) nos recuerda que la tradición ha vinculado a las mujeres con el rol reproductivo y doméstico, mientras que al hombre con el rol productivo, el trabajo comunal y lo público.
} 
En regiones como Centro América, la cultura rural de la población mestiza obliga a que los niños desde que tienen alrededor de 5 o 6 años, vayan conociendo los deberes ligados al rol social asignado, para lograrlo la madre evita que hagan cualquier actividad de las que son consagradas a las mujeres y el padre los va instruyendo poco a poco en el manejo de las especies animales mayores, la producción agrícola y la cacería. En cambio las niñas están limitadas a lo que les enseña la madre, en donde se destaca la cría de especies menores - como aves de corral y cerdos, el cuidado de los huertos familiares, a preparar los alimentos, asear la casa, atender a los varones y comportarse en base a lo que se considera decoroso.

El rol de estas mujeres se engloba principalmente en sus funciones económicas y reproductivas, mientras que su función social como seres humanos que son capaces de construir su propio futuro y contribuir a forjar la historia de sus comunidades, es marginal.

Su función económica tiene por objetivo sostener el hogar en base a la administración de los recursos aportados por sus cónyuges, y en el peor de los casos solo con los generados por ellas; la reproductiva se fundamenta en dar vida y mantenerla, lo que se concretiza con los hijos. En la cultura rural el no tener hijos acarrea una vergüenza moral, y que las mujeres no den a luz niños puede servir de pretexto a su pareja para ser infiel y formar otro hogar, lo que la comunidad no condena, salvo raras excepciones pero desde la cosmovisión de los sectores poblacionales que profesen una religión que no acepte la poligamia.

En lo relacionado a lo social, las mujeres por lo general dependen de lo que diga su pareja o el líder comunitario. Un caso que ilustra en cierta forma esta situación, fue el que viví por más de 4 años en la aldea de la Dalia, ${ }^{4}$ mientras trabajaba con Visión Mundial contribuyendo al desarrollo de varias comunidades rurales; en este lugar la mayoría de las personas que integraban las estructuras organizativas vinculadas a la educación y salud eran mujeres, a las reuniones por lo general solo asistían ellas acompañadas del Presidente del Patronato, quien muy poco hablaba, pero al momento de tomar las decisiones tenían que estar avaladas por él, la pregunta que le hacían era siempre la misma, la cual consistía en la siguiente frase: ¿Y usted qué dice?

La condición de ser mujer cuando la define la cultura patriarcal implica prácticas que exponen a la muerte, entre las que se pueden destacar la mutilación de genitales, lo que afecta aproximadamente a "92 millones de mujeres y niñas de más de 10 años” (Organización Mundial

\footnotetext{
${ }^{4}$ La aldea de la Dalia se localiza en el municipio de Jocón, el que pertenece al departamento de Yoro, Honduras; sus habitantes se dedican a la extracción maderera, al cultivo de especies menores y a la agricultura de subsistencia.
} 
de la Salud 2012: 1) en África. ${ }^{5}$ Y la indiferencia con que son atendidos los femicidios en regiones como Centro América, en donde las cifras proporcionadas por el Consejo Centroamericano de Procuradores de Derechos Humanos (2006: 58-67), muestran que la situación se está agudizando en países como Guatemala, Honduras ${ }^{6}$ y El Salvador.

\section{La precariedad de las mujeres rurales}

Las comunidades rurales al no tener la variedad de actores sociales ni la diversidad cultural que existe en las ciudades, se convierten en espacios con particularidades propias, con una cultura más homogénea, arraigada en usos y costumbres que han sido transmitidos de generación en generación y una mayor vinculación con la naturaleza.

Los roles sociales asignados de acuerdo al género tienen mayor fuerza de coacción sobre las personas, la economía se caracteriza por depender de rubros como las cosechas agrícolas, la cría de animales, el aprovechamiento forestal y la explotación minera. La prestación de los servicios básicos de salud y educación por parte del Estado, son limitados o inexistentes, en especial cuando las comunidades se encuentran más alejadas de la ciudad o pertenecen a minorías étnicas.

La situación de las mujeres rurales a nivel mundial es precaria, los datos proporcionados por el Grupo de Acción Interinstitucional sobre la Mujer Rural ${ }^{7}$ (2014: 1) publicados el 2012, revelan que están en peores condiciones que los hombres rurales y

\footnotetext{
${ }^{5}$ Para tratar de eliminar esta práctica cultural que atenta contra la dignidad de las mujeres, la Asamblea General de las Naciones Unidas (2012: 1) aprobó la resolución 67/146 -el 20 de diciembre de 2012-, denominada "Intensificación de los esfuerzos mundiales para la eliminación de la mutilación genital femenina", la que tendrá valor y efecto una vez que los países la hayan ratificado.

${ }^{6}$ La situación de la violencia contra las mujeres en Honduras es grave, de acuerdo a los datos proporcionados por la Unidad de Género del Observatorio de la Violencia del Instituto Universitario en Democracia, Paz y Seguridad de la Universidad Nacional Autónoma de Honduras (2013: 1), las muertes violentas de mujeres y femicidios, entre el 2005 y el 2012 aumentaron en $246.3 \%$, llegando este último año a 606.

${ }^{7}$ El Grupo de Acción Interinstitucional sobre la Mujer Rural, está dirigido por la Organización de las Naciones Unidas para la Alimentación y la Agricultura (FAO), El Fondo Internacional de Desarrollo Agrícola (FIDA) y el Programa Mundial de Alimentos (PMA), a los que se unen otras dependencias de las Naciones Unidas, vinculadas con los temas de educación, salud, medio ambiente, población, comercio y desarrollo.
} 
que las mujeres y hombres urbanos. En los siguientes párrafos, esbozaré de forma general, su aportación a la economía del hogar, las condiciones en que viven referente a la salud y la educación. ${ }^{8}$

En regiones como el África Subsahariana, en conjunto las mujeres "invierten unas 40,000 millones de horas anuales, proveyendo de agua a ellas y a su familia" (UNIFEM, 2012: 37), aparte de proporcionar los cuidados necesarios a sus hijos, a la pareja y desarrollar otras actividades vinculadas a la economía del hogar. Pero no son remuneradas y su cultura ni considera como un trabajo lo que hacen, a esto Shiva (1997: 85) lo ha denominado una forma de sustento silenciosa pero esencial.

Las mujeres representan el " $43 \%$ de la mano de obra agrícola en los países en desarrollo" (ONU Mujeres 2012: 1), sin embargo, el acceso que tienen a la tierra es limitado, no solo por el costo que alcanza una hectárea en comparación a los recursos económicos que reciben por el trabajo asalariado que desempeñan o la venta de sus productos, a esto se agregan, las "limitaciones legales y culturales" (Grupo de Acción Interinstitucional de la Mujer Rural 2014: 3) a las que se enfrentan en los asuntos relacionados con la herencia familiar.

En las áreas rurales que históricamente han tenido tierras de uso colectivo, en donde las mujeres pueden establecer cultivos de subsistencia, obtener recursos como leña, plantas medicinales y cazar animales, su situación se agrava debido a la privatización y utilización de estas tierras en rubros como la minería, la extracción forestal y los monocultivos a gran escala. Shiva (1998: 107) nos recuerda que la libertad de comercio bajo la cual operan las grandes empresas agrícolas, les niega a las mujeres la libertad de producir, transformar y consumir alimento de acuerdo a las necesidades ambientales, económicas y culturales locales.

En lo referente a la salud, las mujeres se enfrentan a varios desafíos, los más sobresalientes son los que tienen que ver con la educación para el manejo de los

\footnotetext{
${ }^{8}$ Existen otros factores como la violencia contra las mujeres, la aplicación de la justicia, la participación política y la cosmovisión religiosa, que son de utilidad para conocer la situación en que viven las mujeres en las áreas rurales; en mi caso por cuestión de tiempo, me limito a los tres que he seleccionado.
} 
agrotóxicos, la atención a la salud sexual y reproductiva, la nutrición y el acceso a los servicios curativos ofrecidos por el Estado.

El uso y manejo de los agrotóxicos representa un problema serio, ya que "las sustancias químicas tóxicas [...] afectan particularmente al cuerpo femenino" (Puleo 2011: 415); las mujeres se exponen a ellas no solo en las áreas de cultivo, lo hacen también en el hogar, porque los guardan en las habitaciones o la cocina. En países como Honduras, existen familias que usan los recipientes de este tipo de productos, para almacenar agua que después consumen, sin embargo, las capacitaciones sobre la forma en que se deben manejar, se les brinda por lo general solo a los hombres.

A las mujeres culturalmente se les ha obligado a ser las protectoras de la salud de los miembros del hogar, pero sin contar con el poder pleno en la toma de decisiones para hacerlo, lo que les genera un alto costo, que incluso lo pueden pagar con su vida. En El Salvador, ellas son las principales víctimas del VIH/SIDA, Flores (2009: 9) ha documentado, que los temas de salud sexual siguen siendo un tabú en este país, condenados y considerados un pecado desde las estructuras religiosas, a lo que agrega, que el $67 \%$ de las madres que dan a luz por primera vez lo hacen como producto de una violación, que en el 47\% de los casos, ha ocurrido bajo una condición incestuosa.

El sistema patriarcal vulnera el bienestar de las que protegen la vida humana y mantienen una estrecha relación con la naturaleza. "Las mujeres rurales producen gran parte de los alimentos del mundo [...] y viven sin la garantía de una nutrición básica” (KiMoon 2012: 1), participan en el cultivo, administran y preparan los alimentos, pero sirven primero los de mejor calidad a su pareja y a los hijos, mientras ellas consumen lo que sobra.

La educación escolar puede contribuir a superar las desigualdades de género, al dotar a las niñas de la capacidad de leer y escribir, lamentablemente los sistemas educativos tienen una escasa cobertura en las áreas rurales. El Grupo de Acción Interinstitucional de la Mujer Rural (2014: 3) al levantar datos de los hogares de 42 países, identificó que las niñas cuentan con mayor probabilidad de no estar escolarizadas, en comparación a los niños de sus localidades. 


\section{El aporte del ecofeminismo al desarrollo rural sostenible}

Los tres apartados anteriores los he utilizado para definir lo que entiendo por ecofeminismo y desarrollo rural sostenible, reflexionar sobre el rol social de las mujeres rurales y su precariedad tomando de referencia el aporte que hacen a la economía del hogar, así como la situación que viven en lo referente a la salud y la educación. El cuarto apartado lo dedicaré a reflexionar sobre la importancia que tiene el ecofeminismo para impulsar el desarrollo rural sostenible, haciendo énfasis en la dimensión teórica y práctica que integran al mismo.

Desde la dimensión teórica el ecofeminismo ha estructurado un conjunto de ideas que resultan de gran utilidad para generar cambios a nivel de la conciencia. Solo el hecho de intentar desmitificar la percepción de inferioridad que el patriarcado ha creado de las mujeres y la naturaleza, frente a los hombres y la cultura, contribuye a posesionar a las mujeres en la sociedad como constructoras de su propia historia, lo que se puede reconocer en el siguiente planteamiento de Gebara (2000: 19), quien afirma que:

Diferentes grupos de mujeres reivindican hoy el respeto a la integridad de su cuerpo. Rehúsan ser apenas vientre y seno o tan solo sexo genital. Rehúsan ser apenas ayudantes y complementos en la construcción de la historia. Y ese rehusar, de diferentes facetas, parece inaugurar hoy nuevos tipos de relaciones sociales que incluyen avances, contradicciones e interrogantes.

El ideal ecofeminista de "detener y en la medida de lo posible, revertir el deterioro ecológico que sufre el planeta" (Bustillos Durán 2005: 61), y transformar la cultura patriarcal que está expoliando a las mujeres rurales y a la naturaleza, por una cultura del respeto mutuo, en la dimensión práctica representa una gran oportunidad para impulsar el desarrollo rural sostenible. 
En este ideal la transformación del orden social y de la cosmovisión patriarcal imperante es prioritaria, porque influyen de forma directa en la búsqueda de una mayor igualdad de género. Lo que implica mejorar las condiciones materiales de vida, respetar y hacer un uso racional de los recursos naturales, pero también incidir en la interiorización de valores y prácticas culturales inmateriales que dignifiquen a las mujeres.

Las organizaciones ecofeministas cada día ganan mayor protagonismo, muchas de ellas desarrollan su trabajo en los países empobrecidos de América, África y Asia. El ejemplo de movimientos como Chipko de la India, ha seguido inspirando a mujeres y hombres de todo el planeta, que han decidido luchar contra la desigualdad de género, el cambio climático, por la búsqueda de la soberanía alimentaria de los pueblos, y otros problemas que afectan a la mayor parte de la humanidad.

Ecofeminismo y desarrollo rural sostenible son dos términos que en la teoría y en la práctica se encuentran íntimamente ligados, basta con ver la misión que tienen organizaciones como Navdanya, de la India -liderada por Vandana Shiva-, el movimiento Cinturón Verde de Kenia -inspirado en el legado de Wangari Maathai-, o la Fundación Rigoberta Menchú de Guatemala. Todas ellas se enfocan en fomentar la participación de las mujeres y construir junto a los hombres relaciones sociales basadas en la equidad, así como a proteger y hacer un uso responsable de los recursos naturales, para asegurar los medios de vida que necesitan las personas en especial de las áreas rurales.

El valioso aporte del ecofeminismo a la búsqueda del desarrollo sostenible es indiscutible, sin embargo por lo general las organizaciones que lo profesan se han concentrado en las ciudades más grandes y en las comunidades rurales aledañas. Ante lo cual la realidad que viven las mujeres de las comunidades más retiradas de los centros de poder político y económico, también les invita a tenderles la mano, y les recuerda que en estos espacios es donde más se reafirma la necesidad de hacer realidad su propuesta, ya que en ellos se arraiga con mayor fuerza la cultura patriarcal, las mujeres se encuentran en peores condiciones de pobreza y coexisten con las mayores reservas de los recursos 
naturales, que se han convertido en el botín que persiguen las empresas nacionales e internacionales extractoras de materias primas.

\section{Consideraciones finales}

El ecofeminismo es de gran utilidad para impulsar el desarrollo rural sostenible, debido a que revalora la importancia que tiene la mujer y la naturaleza "que trabajan para producir y reproducir la vida [y que] son declaradas improductivas" (Shiva 1995: 84) en el modelo de desarrollo liberal patriarcal.

Las organizaciones ecofeministas se han convertido en uno de los sectores más activos de la sociedad civil de los países empobrecidos, que cooperan con las mujeres $-\mathrm{y}$ los hombres- para mejorar sus condiciones de vida. Sus acciones incluyen la formación política, la defensa y la promoción del uso adecuado de los recursos naturales, el fomento de iniciativas micro-empresariales, el fortalecimiento de las competencias laborales, y cuando es necesario, recurren a la denuncia o movilización para protestar contra lo que consideran un peligro para las mujeres y la naturaleza.

El ecofeminismo como fuente del conocimiento humano, en el marco del desarrollo rural sostenible, debe tener en cuenta dos aspectos, el primero, es que en la búsqueda de la igualdad no hay espacio para el extremismo, porque este puede destruir el ideal que se pretende lograr, el segundo es que se necesita, como lo ha expuesto Calatrava Requena (2002: 85), introducir el tema de género en el discurso político de las comunidades, orientado a generar cambios en su cultura inmaterial, los que se deben manifestar en las prácticas y actitudes de sus habitantes. 


\section{Referencias bibliográficas}

Asamblea General de las Naciones Unidas. 2012. "Intensificación de los esfuerzos mundiales para la eliminación de la mutilación genital femenina". http://www.un.org/es/comun/docs/?symbol=A/RES/67/146 [8 feb. 2014].

Bustillos Durán, Sandra. 2005. "Mujeres de tierra: Ambientalismo, feminismo y ecofeminismo". Nóesis. Género, feminismo(s) y violencia desde la frontera norte 15: 59-77.

Calatrava Requema, Javier. 2002. "Mujer y desarrollo rural en la globalización: De los proyectos asistencialistas a la planificación de género". Revista ICE: Globalización y Mundo Rural, 803: 73-90.

Congreso de la Unión. 2001. Ley de desarrollo rural sustentable. México, D.F: Cámara de Diputados del H. Congreso de la Unión.

Consejo Centroamericano de Procuradores de Derechos Humanos. 2006. Situación y análisis del femicidio en la Región Centroamericana. http://www.cawn.org/espa nol/publicaciones/documentos/otras/Femicidio oficial.pdf [15 feb. 2014].

Field, Leonard. 1997. "Imaginarios, cuentas y una condición básica del desarrollo sostenible". El desarrollo sostenible en el medio rural. Ed. Luciano Martínez. Quito: FLACSO, Sede Ecuador. 31-38. http://www.flacsoandes.edu.ec/libros/ digital/46772.pdf [11 feb. 2014].

Flores, Rosibel. 2009. "Situación de las Mujeres Rurales de El Salvador". Mujer Rural: Boletin elaborado por la Asociación Latinoamericana de Organizaciones de Promoción al Desarrollo A.C. 7-10. http://www.landcoalition.org/sites/default/files/legacy/uploads1/mujer_ruralfinal [11 de feb. 2014].

Gebara, Ivone. 2000. Intuiciones ecofeministas: ensayo para repensar el conocimiento y la religión. Madrid: Editorial Trotta.

Grupo de Acción Interinstitucional de la Mujer Rural. 2014. La mujer rural y los objetivos de desarrollo del milenio. http://www.un.org/womenwatch/feature/rural women/documents/Es-Rural-Women-MDGs-web.pdf [4 feb. 2014].

Ki-Moon, Ban. 2012. Mensaje del Secretario General para el 2012: Día internacional de las mujeres rurales. http://www.un.org/es/events/ruralwomenday/2012/ sgmessage.shtml [6 Feb. 2014]. 
Martín Beristain, Carlos, Darío Páez Rovira e Itziar Fernández. 2009. Las palabras de la selva: Estudio psicosocial del impacto de las explotaciones petroleras de Texaco en las comunidades amazonicas de Ecuador. Bilbao: Lankopi.

Mies, Maria y Vandana Shiva, eds. 1997. “Introducción: ¿Por qué escribimos juntas este libro?”. Ecofeminismo: Teoría, crítica y perspectivas. Barcelona: Icaria editorial. $7-36$.

ONU Mujeres. 2012. El papel de las mujeres en el desarrollo rural, la producción alimentaria y la erradicación de la pobreza. http://www.unwomen.org/es/ne ws/stories/2012/10/the-role-of-women-in-rural-development-food-productionandp overty-eradication [5 feb. 2014].

Organización Mundial de la Salud. 2012. Mutilación genital femenina. Centro de prensa. http://www.who.int/mediacentre/factsheets/fs241/es/ [8 feb. 2014].

Puleo, Alicia H. 2011. Ecofeminismo para otro mundo posible. Madrid, España: Ediciones Cátedra.

. 2013. "El concepto de género como hermenéutica de la sospecha: de la biología a la filosofía moral y política". ARBOR Ciencia, Pensamiento y Cultura Septiembre (189-763): 1-10. doi:http://dx.doi.org/10.3989/arbor.2013.763 n5007 [10 feb. 2014].

Riechmann, Jorge. 2000. Un mundo vulnerable: Ensayo sobre ecología, ética y tecnociencia. Madrid: Catarata.

Shiva, Vandana. 1995. Abrazar la vida: Mujer, ecología y supervivencia. Madrid: Grafistaff.

1997. "El empobrecimiento del medio ambiente: las mujeres y los niños, los últimos". Ecofeminismo: Teoría, crítica y perspectiva. Eds. Maria Mies y Vandana Shiva. Barcelona: Icaria editorial. 107-136

. 1998. "El GATT, la agrícultura y las mujeres del tercer mundo". La praxis del ecofeminismo: Biotecnología, consumo, reproducción”. Eds. Maria Mies y Vandana Shiva. Barcelona: Icaria editorial. 107-128.

Unidad de Género del Observatorio de la Violencia del Instituto Universitario en Democracia, Paz y Seguridad de la Universidad Nacional Autónoma de Honduras. 2013. Observatorio de muertes violentas de mujeres y femicidios: Resultado del análisis enero-diciembre 2012. Tegucigalpa, Honduras. 
UNIFEM. (2012). ¿Quién responde a las mujeres? Género y rendición de cuentas. UNIFEM: Fondo de Desarrollo de las Naciones Unidas para la Mujer. New York. http://www.unifem.org/progress/2008/media/POWW08_Report_Full_Text_es.pdf [28 feb. 2014].

Warren, Karen J. 2003. "Introducción filosofías ecofeministas: Una mirada general”. Filosofías ecofeministas. Eds. Karen J. Warren. Barcelona: Icaria editorial. 11-33. 